\title{
Programmed cell death-1/programmed cell death-ligand 1 inhibitors exert antiapoptosis and antiinflammatory activity in lipopolysaccharide stimulated murine alveolar macrophages
}

\author{
LINGLING JIA ${ }^{1}$, KAI LIU ${ }^{1}$, TENG FEI ${ }^{1}$, QIAN LIU ${ }^{1}$, XIWEI ZHAO ${ }^{1}$, LINYI HOU ${ }^{2}$ and WENKAI ZHANG ${ }^{2}$ \\ ${ }^{1}$ Department of Thoracic Surgery, Shanxi Medical University; ${ }^{2}$ Department of Intensive Care Unit, \\ Second Hospital of Shanxi Medical University, Taiyuan, Shanxi 030001, P.R. China
}

Received January 4, 2020; Accepted June 17, 2020

DOI: $10.3892 /$ etm.2021.9831

\begin{abstract}
Acute lung injury caused by sepsis remains one of the most difficult challenges faced by patients in intensive care units and is associated with a high mortality rate. The aim of the present study was to investigate whether programmed cell death (PD)-1/programmed cell death-ligand 1 (PD-L1) inhibitors reduce alveolar macrophage apoptosis, reduce inflammatory factor release and relieve inflammation. For this purpose, murine alveolar macrophages, $\mathrm{MH}-\mathrm{S}$, were cultured and divided into control, lipopolysaccharide (LPS) and LPS+BMS-1 (PD-1/PD-L1 inhibitors) groups. LPS $(10 \mathrm{ng} / \mathrm{ml})$ was added to the LPS and LPS+BMS-1 groups for $24 \mathrm{~h}$ and PD-1/PD-L1 inhibitor BMS-1 $(1 \mu \mathrm{mol} / \mathrm{l})$ was added to the LPS+BMS-1 group for $72 \mathrm{~h}$. PD-1 mRNA expression was detected using reverse transcription-quantitative PCR and PD-1 protein expression was detected using western blotting in the control, LPS and LPS+BMS-1 groups of macrophages. MH-S apoptosis was detected using flow cytometry with Annexin V/PI staining. The levels of the inflammatory factors interleukin (IL)-1 $\beta$, IL-6, tumor necrosis factor (TNF)- $\alpha$ and IL-10 were detected by ELISA. Murine alveolar macrophages expressed PD-1 at both the molecular and protein levels and PD-1 expression was increased in MH-S cells stimulated with LPS. Compared with the LPS group, the expression of PD-1 in the LPS+BMS-1 group was significantly decreased. Flow cytometry demonstrated that there was increased apoptosis of alveolar macrophages in the LPS group compared with the control group, whereas, alveolar macrophages notably decreased apoptosis in the LPS+BMS-1 group compared with the LPS group. There was no statistical difference between the
\end{abstract}

Correspondence to: Dr Wenkai Zhang, Department of Intensive Care Unit, Second Hospital of Shanxi Medical University, 382 Wuyi Road, Taiyuan, Shanxi 030001, P.R. China

E-mail: 13994206729@163.com

Key words: sepsis, acute lung injury, murine alveolar macrophages, apoptosis, antiinflammation, programmed cell death-1/programmed cell death-ligand 1 inhibitors control group and the LPS+BMS-1 group. IL-1 $\beta$, IL-6, TNF- $\alpha$ and IL-10 were increased in the LPS group compared with the control group. The levels of IL-1 $\beta$, IL- 6 and TNF- $\alpha$ in the LPS+BMS-1 group were lower compared with those in the LPS group whereas IL-10 was further increased. In vitro, the PD-1/PD-L1 inhibitor, BMS-1, decreases alveolar macrophage apoptosis compared with the LPS group to maintain effective immune clearance and reduce inflammatory factor release. This decreased the inflammatory response and reduced acute lung injury caused by sepsis. Therefore, PD-1/PD-L1 inhibitors may be a potential therapeutic target for acute lung injury in patients with sepsis.

\section{Introduction}

Sepsis is a life-threatening disease that leads to organ dysfunction caused by a dysregulated host response to infection (1). According to statistical reports in 2016, 300-1,000 out of 10,000 individuals suffer from sepsis per year in developed countries (2). The lung is one of the primary affected organs (3). Due to a lack of effective treatment methods, acute lung injury (ALI) caused by sepsis is one of the main serious diseases which affect global health (4). Therefore, further investigation into the mechanism of ALI in sepsis is needed to improve disease prognosis and to decrease the mortality rate. The mechanism of ALI is highly complex as its pathophysiological features involve inflammatory cells, inflammatory mediators and the abnormal apoptosis of cells in the lung, including alveolar type II epithelial cells, pulmonary vascular endothelial cells and alveolar macrophages (AMs) (5-8). In sepsis, endotoxin binds to the corresponding receptor on the surface of alveolar macrophages, which induces the release of inflammatory mediators (9). These inflammatory mediators promote the chemotaxis of neutrophils into the lung, leading to further increased release of inflammatory mediators that finally results in damage to the alveolar epithelium and vascular endothelium (10). The disruption of the integrity of the respiratory membrane increases permeability, promotes pulmonary edema, causes ALI and further aggravates acute respiratory distress syndrome (ARDS) (10). A previous study has found that macrophage inhibitory factor is positively associated with the severity of ALI disease due to its presence 
in the serum of patients with sepsis (11). This indicates that proper functioning and maintaining the levels of macrophages will help to reduce the degree of damage caused by sepsis. Therefore, macrophages, including resident AMs and macrophages recruited from the blood, are key factors in the pathogenesis of ALI/ARDS. In addition, the occurrence of $\mathrm{AM}$ apoptosis and dysfunction increases the risk of death in patients with sepsis (12).

Programmed cell death-1 (PD-1) is a type I transmembrane protein of the B7/CD28 superfamily with a relative molecular weight of 50 52.5 kDa that functions as an immunosuppressive molecule (13). In addition, PD-1 contains an immunoreceptor tyrosine inhibitory motif and immunoreceptor tyrosine-based switch motif (ITSM) (14). Various immune cells, including $\mathrm{CD}^{+}{ }^{+}$and $\mathrm{CD}^{+}{ }^{+} \mathrm{T}$ cells, $\mathrm{B}$ cells, natural killer T cells, dendritic cells (DCs) and monocytes/macrophages, can express high levels of PD-1 following stimulation by inflammatory or tumor factors (15). PD-1 has two ligands, programmed cell death-ligand 1 (PD-L1) also known as B7-H1 or CD274 and programmed cell death-ligand 2 PD-L2 also known as B7-DC or CD273. PD-L1 is expressed in several tissue and cell types and PD-L2 is expressed on both DCs and macrophages (15). After PD-1 binds to its corresponding ligand, Src homology 2 domain-containing protein tyrosine (SH)-1 and SHP-2 phosphatase bind to the ITSM inducing the dephosphorylation of downstream $\mathrm{T}$ cell and B cell receptor effector molecules, such as phosphatidylinositol 3-kinase (PI-3K), protein kinase B (AKT/PKB), and extracellular signal-regulated kinase 2 (ERK2) (16-18). Previous studies have reported that the expression of PD-1 on macrophages plays an important role in sepsis-induced ALI/ARDS. For example, knockout of the $p d-1$ gene in mice increased the ability of macrophages to clear bacteria in the blood and peritoneal lavage fluid, as well as reduce the release of the inflammatory factors TNF- $\alpha$, IL-1 $\beta$, IL-10 and C-C motif chemokine ligand 2 (19). Bao et al (20) found that hemorrhagic shock/sepsis increased the expression of PD-L1 in the lung tissue of mice with ALI. In the aforementioned study, damage to the lung tissue of $p d-l l$ gene-deficient ALI mice was mild and the levels of proinflammatory cytokines interleukin (IL) 6 and tumor necrosis factor (TNF) $\alpha$ in the bronchoalveolar lavage fluid were also significantly reduced, which confirmed that PD-L1 may be involved in the immune regulation of ALI by reducing the levels of inflammatory factors.

The aims of the present study were to determine the in vitro expression levels of PD-1 in alveolar macrophages in ALI caused by sepsis, if this phenomenon contributes to the acceleration of the alveolar macrophages apoptosis, and if the decreased secretion of inflammatory factors attenuate the degree of lung damage when the binding of PD-1 and PD-L1 is inhibited. PD-1/PD-L1 inhibitors can inhibit the downstream molecular effects and reduce apoptosis by blocking the binding of PD-1 to PD-L1. In the present study, it was confirmed that BMS-1, a small molecular PD-1/PD-L1 inhibitor, can be used not only for tumor research, but also for research into inflammation (21).

\section{Materials and methods}

Cell culture and treatment. A mouse alveolar macrophage cell line, MH-S, was purchased from Wuhan Punosei Life Technology Co., Ltd. and was routinely passaged in modified
RPMI-1640 medium (Hyclone; GE Healthcare Life Sciences) supplemented with $10 \%$ fetal bovine serum (Cellmax Nutrients BV), $100 \mathrm{U} / \mathrm{ml}$ penicillin and $100 \mu \mathrm{g} / \mathrm{ml}$ streptomycin in a humidified atmosphere of $5 \% \mathrm{CO}_{2}$ at $37^{\circ} \mathrm{C}$. LPS and BMS-1 were at $4^{\circ} \mathrm{C}$. MH-S cells were passaged 3 times and divided into three groups, a control group, LPS group and LPS + BMS-1 group. The control group was provided the same amount of RPMI-1640 medium as the LPS group and LPS + BMS-1 group. When the LPS group and LPS + BMS-1 group cells had grown to $70-80 \%$ confluence, they were stimulated with $10 \mathrm{ng} / \mathrm{ml}$ lipopolysaccharide (LPS; Escherichia coli 055:B5; Sigma-Aldrich; Merck KGaA) for $24 \mathrm{~h}$ in a humidified atmosphere of $5 \% \mathrm{CO}_{2}$ at $37^{\circ} \mathrm{C}$, followed by treatment with $1 \mu \mathrm{mol} / 1 \mathrm{BMS}-1$ for $72 \mathrm{~h}$ at $37^{\circ} \mathrm{C}$. BMS-1 is an inhibitor of the PD-1/PD-L1 protein/protein interaction with an $\mathrm{IC}_{50}$ of 6-100 nM (Fig. 1) (21). It has previously been confirmed that LPS $(0,5,10,20$ or $30 \mathrm{ng} / \mathrm{ml})$ has no toxic effect on MH-S cells and there is no statistically significant difference of different concentrations of LPS on MH-S cells (22). In the present study, MH-S cells were treated with LPS at a concentration of $10 \mathrm{ng} / \mathrm{ml}$ according to a previous study (22).

Reverse transcription-quantitative (RT-q) PCR. Total RNA was extracted from the treated $\mathrm{MH}-\mathrm{S}$ cells $\left(\sim 5 \times 10^{7}\right)$ using TRIzol $^{\circledR}$ reagent (Invitrogen; Thermo Fisher Scientific Inc.). cDNA synthesis was performed from $2 \mu \mathrm{l}$ of total RNA using a RevertAid First Strand cDNA Synthesis kit (Thermo Fisher Scientific Inc.). The reverse transcription protocol was as follows: $37^{\circ} \mathrm{C}$ for $15 \mathrm{~min}$ and heated to $85^{\circ} \mathrm{C}$ for $5 \mathrm{sec}$ to eliminate reverse transcriptase activity. The treated $\mathrm{MH}-\mathrm{S}$ cells were denatured at $95^{\circ} \mathrm{C}$ for $30 \mathrm{sec}$, annealed at $95^{\circ} \mathrm{C}$ for $5 \mathrm{sec}$ and extended at $60^{\circ} \mathrm{C}$ for $30 \mathrm{sec}$, a total of 45 cycles. The levels of PD-1 mRNA expression were assessed using a SYBR Green Mastermix kit (Takara Bio, Inc.) with the CFX96 Touch ${ }^{\mathrm{TM}}$ Real-Time PCR Detection system (Bio-Rad Laboratories, Inc.). The levels of mRNA expression were calculated using the $2^{-\Delta \Delta \mathrm{Cq}}$ method and normalized to GAPDH (23). The primer sequences used are as follows: Mouse PD-1, forward, 5'-ATGACTTCC ACATGAACATCCT-3', reverse, 5'-CTCCAGGATTCTCTC TGTTACC-3'; and GAPDH, forward, GGCAAGTTCAAC GGCACAGT, reverse, ATGACATACTCAGCACCGGG.

Western blotting. Following the different experimental treatments, the MH-S cells $\left(\sim 5 \times 10^{7}\right)$ cultured in 6 -well plates, were harvested and lysed in RIPA lysis buffer (Boster Biological Technology) containing a $1 \%$ protease inhibitor (Boster Biological Technology). The cellular supernatant was collected by centrifugation at $12,000 \mathrm{x}$ g for $15 \mathrm{~min}$ at $4^{\circ} \mathrm{C}$ and the protein concentrations were measured with a bicinchoninic protein assay kit (Bio-Rad Laboratories, Inc.). The protein samples $(20 \mu \mathrm{g} /$ lane $)$ were separated by $12 \%$ sodium dodecyl sulfate-polyacrylamide gel electrophoresis (SDS-PAGE) and transferred onto polyvinylidene difluoride (PVDF) membranes (Zhangshu Zhenghe Biotechnology Co., Ltd.). The membranes were blocked in $5 \%$ non-fat milk proteins for $2 \mathrm{~h}$ at room temperature and then probed with the following primary antibodies: PD-1 (cat. no. MB9410; 1:500; Bioworld Technology Inc.) and $\beta$-actin (cat. no. BS6007; 1:3,000; Bioworld Technology Inc.) overnight at $4^{\circ} \mathrm{C}$. The membranes were subsequently incubated with horseradish peroxidase-conjugated secondary 


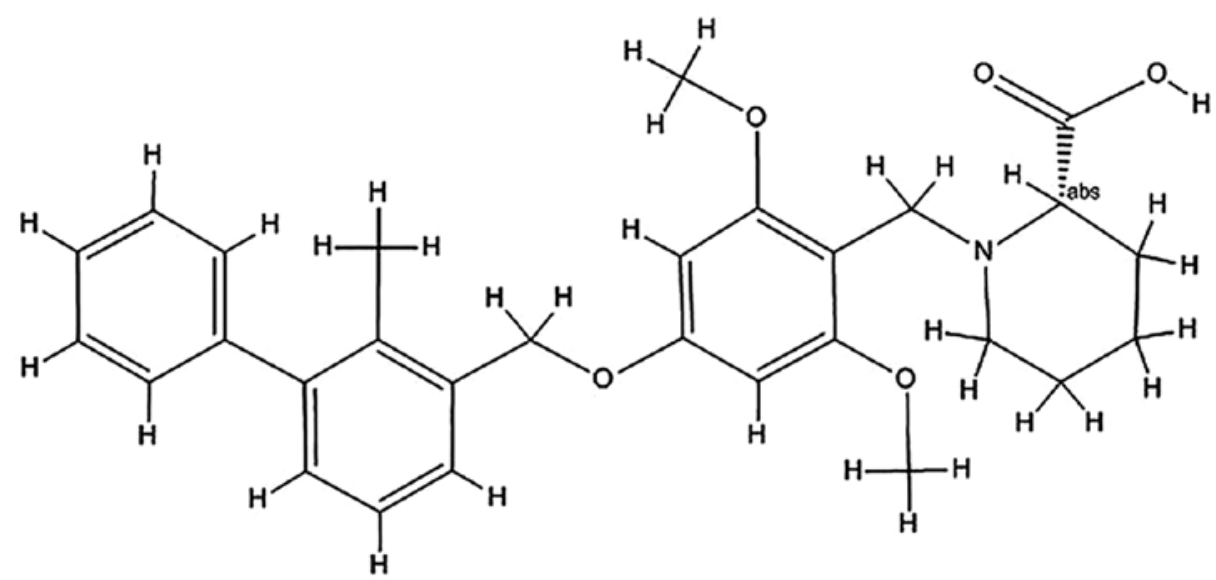

Figure 1. The chemical structure of BMS-1. BMS-1, a small-molecule immune checkpoint inhibitor of PD-1/PD-L1, can inhibit PD-1/PD-L1 protein/protein interaction $\left(\mathrm{IC}_{50}\right.$ between 6-100 $\left.\mathrm{nM}\right)$.

goat anti-mouse antibodies (cat. no. BA1038; 1:1,000; Boster Biology Technology) for $1 \mathrm{~h}$ at $37^{\circ} \mathrm{C}$. Finally, the protein bands were visualized using an enhanced chemiluminescence (ECL) system (Thermo Fisher Scientific, Inc.) and analyzed by ImageJ 1.50 (National Institutes of Health.). $\beta$-actin was used as the loading control.

Flow cytometry. MH-S cells $\left(\sim 5 \times 10^{7}\right)$ cultured in 6-well plates, were harvested and washed three times with PBS and stained with $5 \mu \mathrm{l}$ Annexin V-FITC and $10 \mu \mathrm{l}$ of propidium iodide for $15 \mathrm{~min}$ in $500 \mathrm{ml}$ binding buffer at room temperature. Surface exposure of phosphatidylserine in the apoptotic cells was measured using an Annexin V-FITC Apoptosis Detection kit (Shanghai Gensheng Biotechnology Co., Ltd.) according to the manufacturer's instructions. Apoptosis was analyzed using a flow cytometer (BD FACSCalibur Cell Sorting System; BD Bioscience).

ELISA. Concentrations of IL-1 $\beta$, IL-6, TNF- $\alpha$ and IL-10 in the supernatants of treated $\mathrm{MH}-\mathrm{S}$ cells were measured using respective specific ELISA kits (R\&D Systems, Inc.) in accordance with the manufacturer's instructions (IL-1 $\beta$, mouse IL-1 $\beta$, PicoKine ELISA kit, cat. no. EK0394; IL-6, mouse IL-6, PicoKine ELISA kit, cat. no. EK0411; TNF- $\alpha$, mouse TNF- $\alpha$ PicoKine ELISA kit, cat. no. EK0527; IL-10, mouse IL-10 PicoKine ELISA kit, cat. no. EK0417; all supplied from Boster Biological Technology, Ltd.).

Statistical analysis. Statistical analysis was performed using GraphPad Prism version 7.0 (GraphPad Software, Inc.). The measurement data are presented as the means \pm standard deviation of three independent experiments performed in triplicate. The comparison between the 3 different groups was performed by one-way ANOVA followed by the post hoc Student Newman Keule test. $\mathrm{P}<0.05$ was considered to indicate a statistically significant difference.

\section{Results}

BMS-1 inhibits LPS-induced expression of PD-1 $\mathrm{mRNA}$ and protein in $\mathrm{MH}-\mathrm{S}$ cells. To confirm and explore the effects of

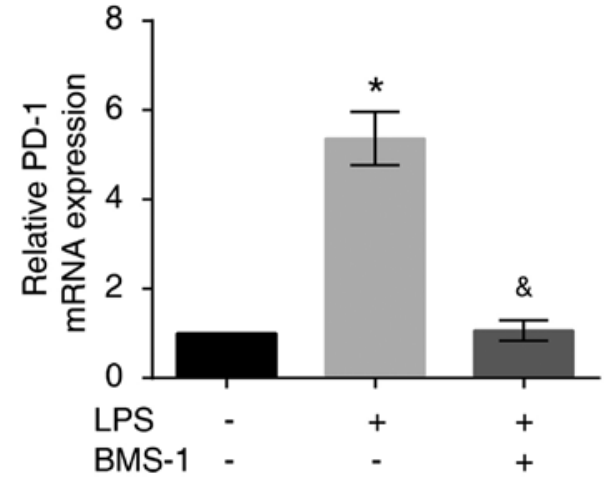

Figure 2. Effects of BMS-1 on LPS-induced expression of PD-1 mRNA in MH-S cells. MH-S cells were stimulated using $10 \mathrm{ng} / \mathrm{ml}$ LPS for $24 \mathrm{~h}$ followed by treatment with $1 \mu \mathrm{mol} / 1 \mathrm{BMS}-1$ for $72 \mathrm{~h}$. The levels of mRNA expression in the treated $\mathrm{MH}-\mathrm{S}$ cells were determined using reverse transcription-quantitative PCR. Data are expressed as the mean \pm standard deviation of three independent experiments performed in triplicate. ${ }^{*} \mathrm{P}<0.05$, LPS group vs. control group; ${ }^{\circledR} \mathrm{P}<0.05$, LPS+BMS-1 group vs. LPS group. LPS, lipopolysaccharide; PD-1, programmed cell death-1.

BMS-1 on LPS-induced MH-S cells, PD-1 mRNA and protein expression were assessed using RT-qPCR and western blotting, respectively. LPS-stimulated MH-S cells had increased levels of PD-1 mRNA and protein expression compared with the control group (Figs. 2 and 3, P<0.01). PD-1 mRNA and protein expression were reduced in $\mathrm{MH}-\mathrm{S}$ cells treated with LPS+BMS-1 compared with cells just treated with LPS (Figs. 2 and 3, $\mathrm{P}<0.01$ ). Taken together, these results demonstrated that BMS-1 inhibited LPS-induced expression of PD-1 in $\mathrm{MH}-\mathrm{S}$ cells.

BMS-1 suppresses LPS-induced apoptosis in MH-S cells. To investigate the apoptotic effect of BMS-1 in LPS-stimulated $\mathrm{MH}-\mathrm{S}$ cells, flow cytometry was performed and the number of apoptotic cells were counted. LPS exposure resulted in enhanced apoptosis compared with the control group $(\mathrm{P}<0.05)$, which was attenuated by BMS-1 treatment (Fig. 4, $\mathrm{P}<0.05)$. These results demonstrated that following LPS stimulation, alveolar macrophages exhibit high levels of PD-1 expression and increased apoptosis. Following treatment with 

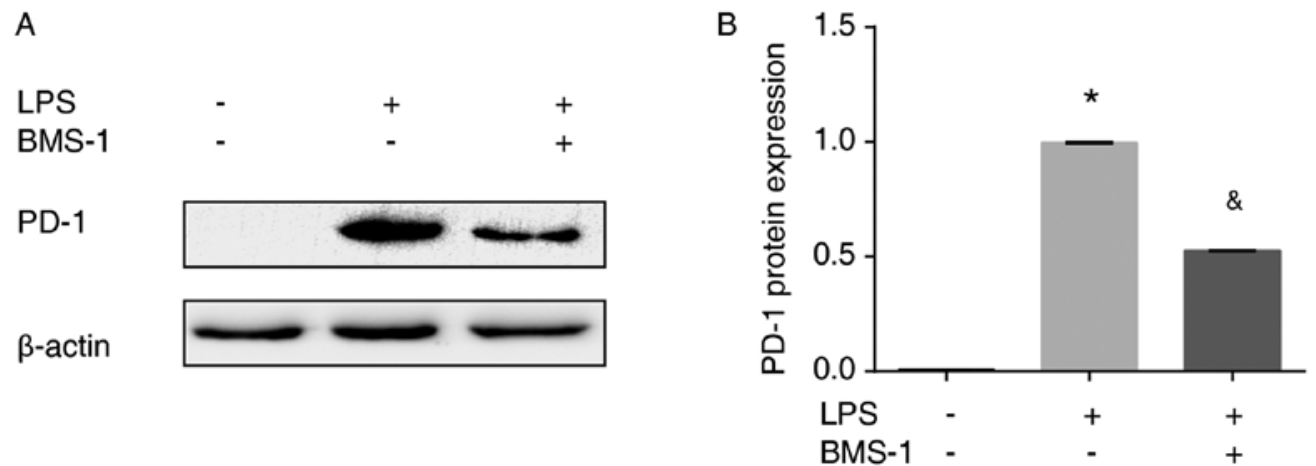

Figure 3. Effects of BMS-1 on LPS-induced expression of PD-1 protein in MH-S cells. (A) Western Blot for relative proteins expressions of PD-1 in three groups. (B) The quantified results of PD-1 protein for figure A MH-S cells were stimulated using $10 \mathrm{ng} / \mathrm{ml} \mathrm{LPS}$ for $24 \mathrm{~h}$ followed by treatment with $1 \mu$ mol/1 BMS-1 for $72 \mathrm{~h}$. The levels of PD-1 protein expression in the treated MH-S cells were determined by western blotting. Data are expressed as the mean \pm standard deviation of three independent experiments performed in triplicate. ${ }^{*} \mathrm{P}<0.05$, LPS group vs. control group; ${ }^{\circledR} \mathrm{P}<0.05$, LPS+BMS-1 group vs. LPS group. LPS, lipopolysaccharide; PD-1, programmed cell death-1.
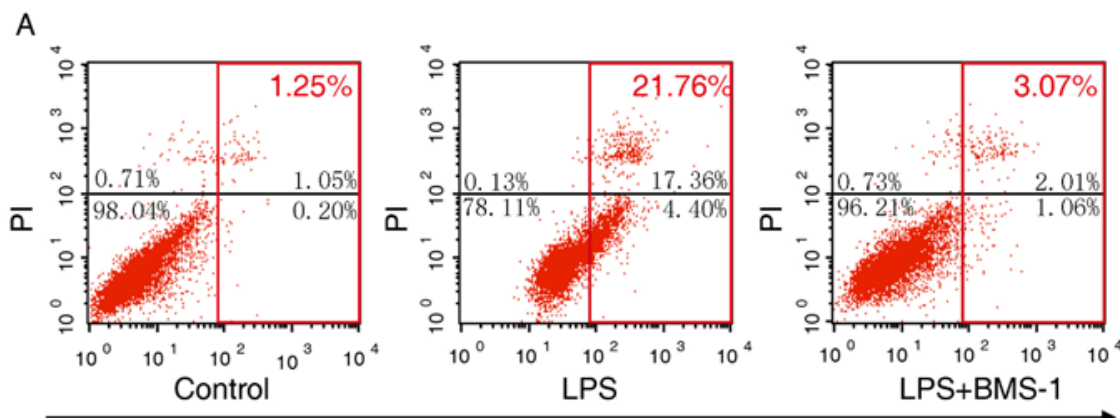

Annexin V-FITC

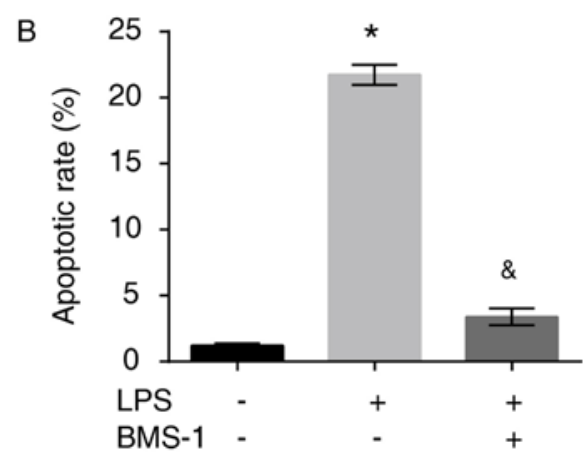

Figure 4. Effect of BMS-1 on LPS-induced apoptosis of MH-S cells. (A) The apoptosis rate of MH-S cells in the three groups.(B) The quantified results of apoptosis rate of MH-S cells for figure A. MH-S cells were stimulated by $10 \mathrm{ng} / \mathrm{ml}$ LPS for $24 \mathrm{~h}$, followed by treatment with $1 \mu \mathrm{mol} / 1 \mathrm{BMS}-1$ for $72 \mathrm{~h}$. The rate of apoptosis in the treated MH-S cells was determined using flow cytometry. Data are expressed as the mean \pm standard deviation of 3 independent experiments performed in triplicate. ${ }^{\text {P }}<0.05$ vs. control group; ${ }^{\circledR} \mathrm{P}<0.05$ vs. LPS group. LPS, lipopolysaccharide; PD-1, programmed cell death-1.

a PD-1/PD-L1 inhibitor, both PD-1 expression and alveolar macrophage apoptosis were decreased.

BMS-1 exerts antiinflammatory activity in LPS-treated $M H-S$ cells. To investigate the antiinflammatory effects of BMS-1, the levels of inflammatory factors IL-1 $\beta$, IL- 6 , TNF- $\alpha$ and IL-10 secreted by LPS-stimulated MH-S cells were detected using ELISA. The secretion of the proinflammatory cytokines IL-1 $\beta$, IL- 6 , TNF- $\alpha$ and antiinflammatory cytokine IL-10 was increased following LPS stimulation (Fig. 5A-D, P<0.05). Treatment with BMS-1 reduced the LPS-induced increase of TNF- $\alpha$, IL-1 $\beta$ and IL- 6 in MH-S cells, while IL-10 was increased significantly (Fig. $5 \mathrm{~A}-\mathrm{D}, \mathrm{P}=0.085$ for $\mathrm{IL}-1 \beta, \mathrm{P}<0.05$ for IL-6 TNF- $\alpha$ and IL-10).

\section{Discussion}

ALI caused by sepsis and ARDS remains the primary cause of death among patients in the ICU (24). Alveolar macrophages serve an important role in the pathophysiological process of ALI (25). In addition, a recent study confirmed that the inhibition of alveolar macrophage apoptosis and excessive inflammatory response can reduce the degree of ALI caused by sepsis (26). The present study investigated the apoptosis of MH-S cells and levels of inflammatory mediators, such as IL-1, IL-6, TNF- $\alpha$ and IL-10, following the administration of the PD-1/PD-L1 inhibitor BMS-1. RT-qPCR and western blotting results confirmed that $\mathrm{PD}-1$ was moderately transcribed and expressed or expressed at basal levels in normal cells; however, 
A
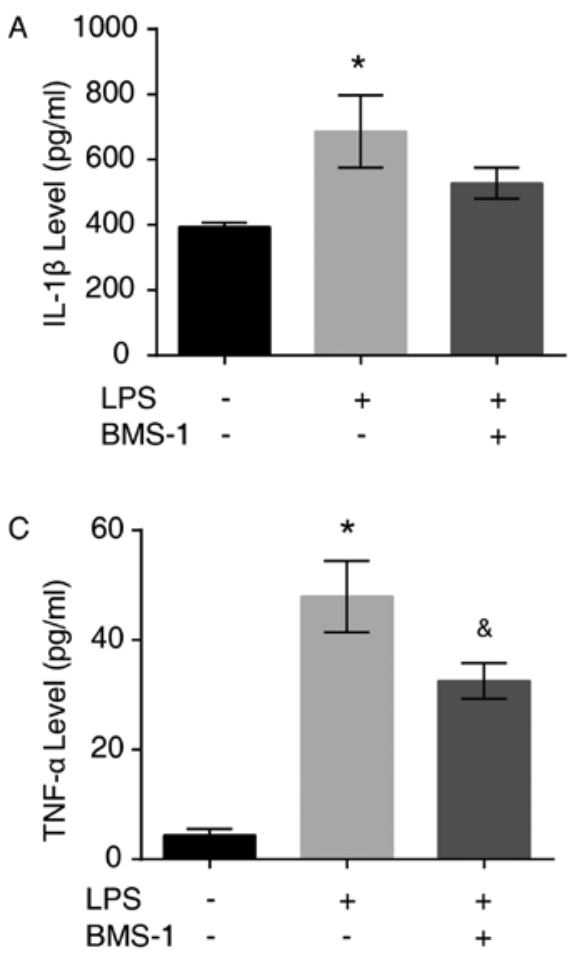

B
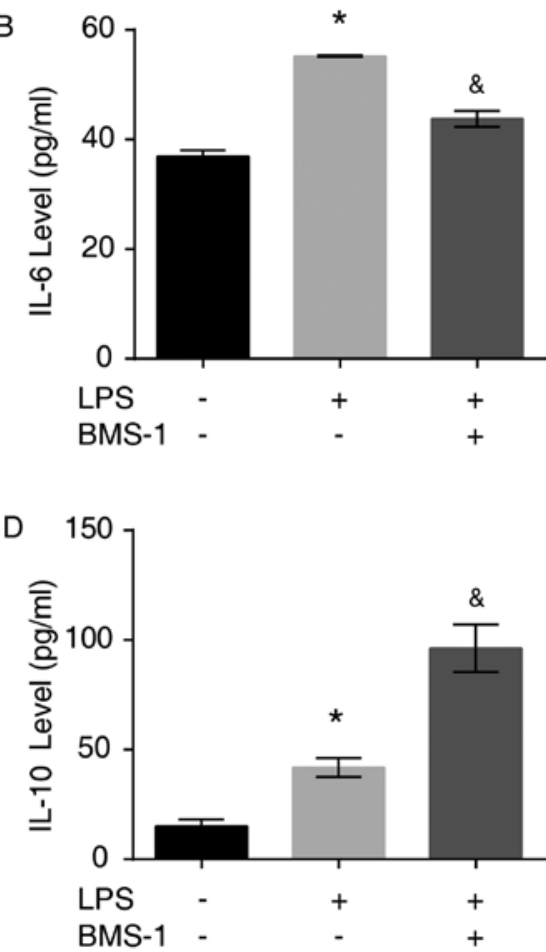

Figure 5. Effects of BMS-1 on LPS-induced production of proinflammatory and antiinflammatory cytokines in MH-S cells. (A) Expression of IL-1 $\beta$ in control group, LPS group and LPS+BMS-1 group. (B) Expression of IL-6 in three groups. (C) Expression of TNF- $\alpha$ in three groups. (D) Expression of IL-10 in three groups. MH-S cells were stimulated by $10 \mathrm{ng} / \mathrm{ml}$ LPS for $24 \mathrm{~h}$ followed by treatment with $1 \mu \mathrm{mol} / 1 \mathrm{BMS}-1$ for $72 \mathrm{~h}$. The concentrations of IL-1 $\beta$, IL-6, TNF- $\alpha$ and IL-10 in the supernatants of the treated MH-S cells were measured using ELISA. The data are expressed as the mean \pm standard deviation of three independent experiments performed in triplicate. "P $<0.05$, LPS group vs. control group; ${ }^{\circledR} \mathrm{P}<0.05$, LPS+BMS-1 group vs. the LPS group. LPS, lipopolysaccharide; PD-1, programmed cell death-1; IL, interleukin; TNF, tumor necrosis factor.

following LPS stimulation, alveolar macrophages expressed high levels of PD-1. The flow cytometry findings of the present study demonstrated that the apoptosis of alveolar macrophages was significantly increased in the LPS group compared with the control group. These findings indicated that PD-1 could be expressed at high levels in alveolar macrophages in ALI, which may cause an increase in the apoptosis of alveolar macrophages. According to the findings of the present study, when PD-1 was bound to its ligand, PD-L1 was inhibited, the levels of PD-1 mRNA and protein expression in the LPS+BMS-1 group were significantly lower and the apoptosis of MH-S cells was decreased compared with the LPS group. The ELISA results demonstrated that the expression of the proinflammatory cytokines IL- $1 \beta$, IL- 6 and TNF- $\alpha$ were significantly increased in the LPS group compared with the control group. In addition, the expression of the antiinflammatory factor IL-10 was markedly increased upon the stimulation of BMS-1, whereas PD-lexpression was inhibited. Following inhibition of PD-1/PD-L1 using BMS-1 in the present study, the levels of proinflammatory cytokines IL-1 $\beta$, IL- 6 and TNF- $\alpha$ decreased significantly and the level of the antiinflammatory cytokine IL-10 increased compared with the LPS treated group. Thus, BMS-1 can inhibit proinflammatory factors and promote the expression of antiinflammatory factors, thereby reducing the inflammatory response associated with ALI.

The present study has several limitations. It has only been shown in vitro that treatment with a PD-1/PD-L1 inhibitor can decrease the apoptosis of alveolar macrophages, reduce inflammatory factor expression. In addition, the study did not investigate if these effects were long lasting. The findings of the present study were only assessed in alveolar macrophage MH-S cells. In addition, the specific mechanism by which PD-1 and PD-1/PD-L1 inhibitors impact the apoptosis of alveolar macrophages and the release of inflammatory factors remains unclear.

A previous study demonstrated that the increase in alveolar macrophages led to an increase in the secretion of proinflammatory mediators, causing chemotactic central granulocytes to accumulate in the lungs, which destroyed alveolar epithelial cells and endothelial cells, resulting in increased permeability and acute pulmonary edema (27). Recently, a study has found that LPS increases PD-1 expression through the NF- $\kappa \mathrm{B}$ signaling pathway (28). This study demonstrated that PD-1/PD-L1 inhibitor, BMS-1 reduced alveolar macrophage apoptosis and decreased the release of inflammatory factors, leading to the question of whether the treatment with BMS-1 has an effect on the polarization of alveolar macrophages. Alveolar macrophages can be divided into two subclasses: M1 and M2 (29). M1s primarily serve a proinflammatory role and are involved in immune responses to bacterial and viral infections. M1 macrophages have high surface CD11c and CD16 expression. M2 macrophages are mainly involved in the antiinflammatory response, which is associated with parasitic infection, tissue remodeling, fibrosis and tumor development, and exhibit high levels of CD206 and arginase 1 surface expression (30). M2 macrophages are further divided into 3 subtypes: A, b and c. Type a is induced by IL- 4 and IL-13, type $b$ is induced by immune complexes 
and toll like receptors or the IL-1 receptor and type $\mathrm{c}$ is induced by IL-10 and glucocorticoids (30). In addition, it has been confirmed that an increase in M1 alveolar macrophages leads to an increase in inflammatory factors and an aggravation of lung injury in rats (31). Thus, inhibiting the polarization of alveolar macrophages to M1 minimizes lung injury (31). Therefore, it can be speculated that PD-1/PD-L1 inhibitors play a balancing role in the polarization of M1 and M2 alveolar macrophages. The antiapoptotic and antiinflammatory efficacy of PD-1/PD-L1 inhibitors in vivo should be assessed in future studies. Further in vitro studies demonstrating whether such PD-1/PD-L1 inhibitors polarize alveolar macrophages need to be conducted.

To the best of our knowledge, the present study is the first to demonstrate that BMS-1, an inhibitor of the PD-1/PD-L1, pathway exerts antiapoptotic and antiinflammatory effects on LPS-stimulated MH-S cells. The findings may provide novel insight into the effects and molecular mechanisms of a PD-1/PD-L1 inhibitor in sepsis-induced ALI. Thus, a PD-1/PD-L1 inhibitor may be a promising therapeutic strategy for treating patients with ALI. Therefore, sepsis-induced ALI animal models are required to further evaluate the effects of a PD-1/PD-L1 inhibitor in vivo.

\section{Acknowledgements}

Not applicable.

\section{Funding}

The present study was supported by grants from the Scientific Research Funding Project for Returnees in Shanxi Province of China (grant no. 2011-105) and the Taiyuan Science and Technology Project of Shanxi Province of China (grant no. 12016905).

\section{Availability of data and materials}

The datasets used and/or analyzed during the current study are are available from the corresponding author on reasonable request.

\section{Authors' contributions}

LJ designed the experiments, performed the reverse transcription-quantitative PCR and western blotting experiments, analyzed the data and wrote the manuscript. WZ and LH designed the experiments and revised the manuscript for important intellectual content. KL and TF cultured the cells and performed flow cytometric analysis. QL and XZ assisted in completing western blot analysis. All authors have read and approved the final manuscript.

\section{Ethics approval and consent to participate}

Not applicable.

\section{Patient consent for publication}

Not applicable.

\section{Competing interests}

The authors declare that they have no competing interests.

\section{References}

1. Singer M, Deutschman CS, Seymour CW, Shankar-Hari M, Annane D, Bauer M, Bellomo R, Bernard GR, Chiche JD, Coopersmith CM, et al: The third international consensus definitions for sepsis and septic shock (Sepsis-3). JAMA 315: 801-810, 2016.

2. Kempker JA and Martin GS: The changing epidemiology and definitions of sepsis. Clin Chest Med 37: 165-179, 2016.

3. Wang YM, Ji R, Chen WW, Huang SW, Zheng YJ, Yang ZT, Qu HP, Chen H, Mao EQ, Chen Y and Chen EZ: Paclitaxel alleviated sepsis-induced acute lung injury by activating MUC1 and suppressing TLR-4/NF- $\kappa$ B pathway. Drug Des Devel Ther 13: 3391-3404, 2019.

4. Johnson ER and Matthay MA: Acute lung injury: Epidemiology, pathogenesis, and treatment. J Aerosol Med Pulm Drug Deliv 23: 243-252, 2010.

5. Zahar JR, Timsit JF, Garrouste-Orgeas M, Français A, Vesin A, Descorps-Declere A, Dubois Y, Souweine B, Haouache H, Goldgran-Toledano D, et al: Outcomes in severe sepsis and patients with septic shock: Pathogen species and infection sites are not associated with mortality. Crit Care Med 39: 1886-1895, 2011.

6. Matthay MA, Ware LB and Zimmerman GA: The acute respiratory distress syndrome. J Clin Invest 122: 2731-2740, 2012.

7. Huang C, Zheng H, He W, Lu G, Li X, Deng Y and Zeng M: Ghrelin ameliorates the human alveolar epithelial A549 cell apoptosis induced by lipopolysaccharide. Biochem Biophys Res Commun 474: 83-90, 2016.

8. Gill SE, Rohan M and Mehta S: Role of pulmonary microvascular endothelial cell apoptosis in murine sepsis-induced lung injury in vivo. Respir Res 16: 109, 2015.

9. Li B, Fang J, Zuo Z, Yin S, He T, Yang M, Deng J, Shen L, Ma X, Yu S, et al: Activation of porcine alveolar macrophages by actinobacillus pleuropneumoniae lipopolysaccharide via the toll-like receptor 4/NF- $\mathrm{KB}$-mediated pathway. Infect Immun 86: e00642-17, 2018.

10. Moldoveanu B, Otmishi P, Jani P, Walker J, Sarmiento X, Guardiola J, Saad M and Yu J: Inflammatory mechanisms in the lung. J Inflamm Res 2: 1-11, 2009.

11. Pahuja M, Tran C, Wang H and Yin K: Alveolar macrophage suppression in sepsis is associated with high mobility group box 1 transmigration. Shock 29: 754-760, 2008.

12. Yamazaki T, Akiba H, Iwai H, Matsuda H, Aoki M, Tanno Y, Shin T, Tsuchiya H, Pardoll DM, Okumura K, et al: Expression of programmed death 1 ligands by murine T cells and APC. J Immunol 169: 5538-5545, 2002.

13. Keir ME, Butte MJ, Freeman GJ and Sharpe AH: PD-1 and its ligands in tolerance and immunity. Annu Rev Immunol 26: 677-704, 2008.

14. Shinohara T, Taniwaki M, Ishida Y, Kawaichi M and Honjo T: Structure and chromosomal localization of the human PD-1 gene (PDCD1). Genomics 23: 704-706, 1994.

15. Hassan SS, Akram M, King EC, Dockrell HM and Cliff JM: PD-1, PD-L1 and PD-L2 gene expression on T-cells and natural killer cells declines in conjunction with a reduction in PD-1 protein during the intensive phase of tuberculosis treatment. PLoS One 10: e0137646, 2015.

16. Dong H, Zhu G, Tamada K and Chen L: B7-H1, a third member of the B7 family, co-stimulates T-cell proliferation and interleukin-10 secretion. Nat Med 5: 1365-1369, 1999.

17. Latchman Y, Wood CR, Chernova T, Chaudhary D, Borde M, Chernova I, Iwai Y, Long AJ, Brown JA, Nunes R, et al: PD-L2 is a second ligand for PD-1 and inhibits $\mathrm{T}$ cell activation. Nat Immunol 2: 261-268, 2001.

18. Taube JM, Klein A, Brahmer JR, Xu H, Pan X, Kim JH, Chen L, Pardoll DM, Topalian SL and Anders RA: Association of PD-1, PD-1 ligands, and other features of the tumor immune microenvironment with response to anti-PD-1 therapy. Clin Cancer Res 20: 5064-5074, 2014.

19. Huang X, Venet F, Wang YL, Lepape A, Yuan Z, Chen Y, Swan R, Kherouf H, Monneret G, Chung CS and Ayala A: PD-1 expression by macrophages plays a pathologic role in altering microbial clearance and the innate inflammatory response to sepsis. Proc Natl Acad Sci USA 106: 6303-6308, 2009. 
20. Bao X, Sun H and Yang Q: The role and mechanism of costimulatory molecular programmed death ligand-1 in acute lung injury. Zhonghua Crisis Emerg Med 28: 498-503, 2016.

21. Zhao Y, Jia Y, Shi T, Wang W, Shao D, Zheng X, Sun M, He K and Chen L: Depression promotes hepatocellular carcinoma progression through a glucocorticoids mediated up-regulation of PD-1 expression in tumor infiltrating NK cells. Carcinogenesis: Feb 4, 2019 (Epub ahead of print). doi: 10.1093/carcin/bgz017.

22. Zhou Z, Su Y and Fa XE: Isorhynchophylline exerts anti-inflammatory and anti-oxidative activities in LPS-stimulated murine alveolar macrophages. Life Sci 223: 137-145, 2019.

23. Livak KJ and Schmittgen TD: Analysis of relative gene expression data using real-time quantitative PCR and the 2(-Delta Delta C(T)) method. Methods 25: 402-408, 2001.

24. Niesler U, Palmer A, Radermacher P and Huber-Lang MS: Role of alveolar macrophages in the inflammatory response after trauma. Shock 42: 3-10, 2014.

25. Kubota Y, Iwasaki Y, Harada H, Yokomura I, Ueda M, Hashimoto $S$ and Nakagawa M: Role of alveolar macrophages in Candida-induced acute lung injury. Clin Diagn Lab Immunol 8: 1258-1262, 2001.

26. Yang L, Zhang Z, Zhuo Y, Cui L, Li C, Li D, Zhang S, Cui N, Wang $\mathrm{X}$ and Gao $\mathrm{H}$ : Resveratrol alleviates sepsis-induced acute lung injury by suppressing inflammation and apoptosis of alveolar macrophage cells. Am J Transl Res 10: 1961-1975, 2018.
27. Fu Y, Zhou J, Liu K, Hou L and Zhang W: Effects of miR-155 on IL-6, IL-10 and MIP-2 in alveolar macrophages of acute lung injury induced by sepsis. Chin J Crit Care Med 5: 159-164, 2019.

28. Nam S, Lee A, Lim J and Lim JS: Analysis of the expression and regulation of PD-1 protein on the surface of myeloid-derived suppressor cells (MDSCs). Biomol Ther (Seoul) 27: 63-70, 2019.

29. Mitsi E, Kamng'ona R, Rylance J, Solórzano C, Jesus Reiné J, Mwandumba HC, Ferreira DM and Jambo KC: Human alveolar macrophages predominately express combined classical M1 and M2 surface markers in steady state. Respir Res 19: 66, 2018.

30. Italiani $\mathrm{P}$ and Boraschi D: From monocytes to M1/M2 macrophages: Phenotypical vs functional differentiation. Front Immunol 5: 514, 2014.

31. Yin D, Wang W, Han W and Fan C: Targeting Notch-activated M1 macrophages attenuate lung tissue damage in a rat model of ventilator induced lung injury. Int J Mol Med 44: 1388-1398, 2019.

This work is licensed under a Creative Commons Attribution-NonCommercial-NoDerivatives 4.0 International (CC BY-NC-ND 4.0) License. 